\title{
Reply to: Comments on "Survival impact of adjuvant chemotherapy in patients with stage IIA colon cancer: Analysis of the National Cancer Database"
}

Dear editor,

We would like to thank our colleague for the thoughtful comment on our study. ${ }^{1}$ Inverse probability of treatment weighting was utilized to balance the two groups (adjuvant chemotherapy vs observation) with respect to the clinically most relevant variables available in the National Cancer Database (NCDB), including age, sex, Charlson Deyo Score, race, high-risk features, facility type, urban/ rural location, education status, insurance status, tumor location, tumor size, year of diagnosis, and lymph node sampling (0-11 vs $12+)$.

We agree that performance status (PS), which unfortunately is not reported in NCDB, represents an uncontrolled confounder as a central factor in cancer care, ${ }^{2}$ not only directly related to prognosis but also commonly used in the decision-making process of a potential benefit of adjuvant chemotherapy. ${ }^{3}$ For these reasons, we acknowledge that patient death in the first months due to poor PS and related morbidity may have contributed to an overestimation of the benefit of adjuvant chemotherapy. ${ }^{4}$

In order to face this limitation, we performed an additional analysis excluding patients who died within 90 days, partially reducing the issue of guarantee-time bias. This separate analysis showed a decreased 5-year survival benefit of $6 \%$ in the adjuvant chemotherapy group (80.0\% [95\% confidence interval $[\mathrm{Cl}]=79.4-80.5]$ ) compared to the observation group $(74.0 \%[95 \% \mathrm{Cl}=73.4-74.5], P<.001)$ (Figure 1A) while a 5 -year survival benefit of $7.3 \%$ was found among high-risk patients $(78.4 \%[95 \% \mathrm{Cl}=77.6-79.3]$ vs $71.1 \%$ [95\% $\mathrm{Cl}=70.1-72.1], P<.001$ ) (Figure 1B). However, these results still suggest a potential survival benefit in selected patients with stage IIA disease and high-risk features undergoing adjuvant chemotherapy.

\section{CONFLICT OF INTEREST}

The authors declare no potential conflict of interest.

\section{DATA AVAILABILITY STATEMENT}

Because of the sensitive nature of the data collected for this study, requests to access the data set from qualified researchers trained in human subject confidentiality protocols may be sent to the author (David W. Larson).

Pietro Achilli (D) Jacopo Crippa
(A)

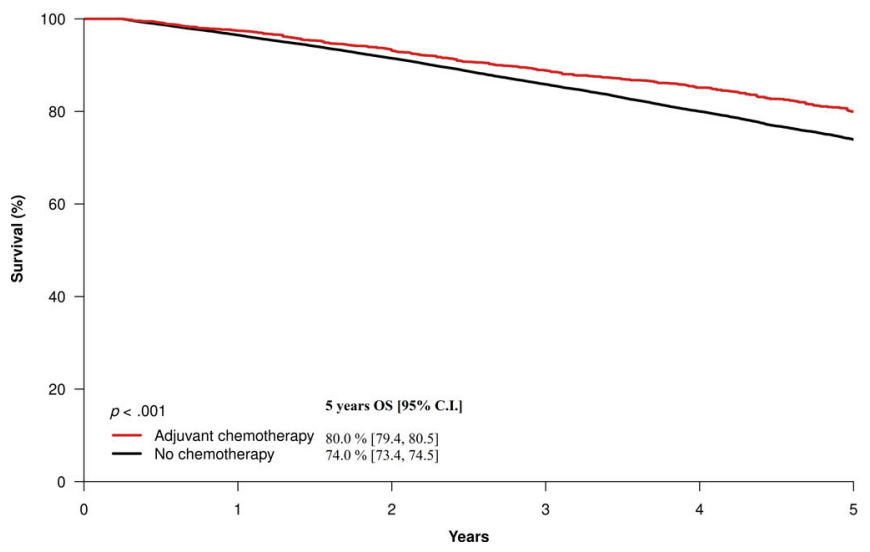

(B)

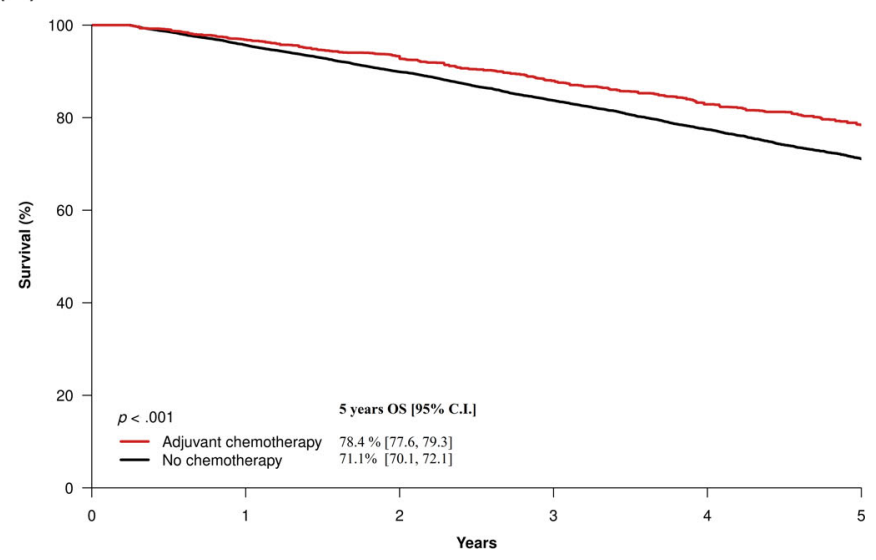

FIGURE 1 Inverse probability of treatment weighting-adjusted Kaplan-Meier analysis of overall survival after the exclusion of death within the first 90 postoperative days. A, Overall-IPTW adjusted. B, High-risk patients-IPTW adjusted. IPTW, inverse probability of treatment weighting [Color figure can be viewed at wileyonlinelibrary.com] 
Fabian Grass

David W. Larson

Colon and Rectal Surgery, Mayo Clinic, Rochester, Minnesota

Correspondence

Pietro Achilli, Mayo Clinic, 200 First Street SW, Rochester, MN

55905.

Email: achilli.pietro@mayo.edu

\section{ORCID}

Pietro Achilli (D) https://orcid.org/0000-0001-9021-9521

\section{REFERENCES}

1. Achilli P, Crippa J, Grass F, et al. Survival impact of adjuvant chemotherapy in patients with stage IIA colon cancer: analysis of the National Cancer Database [published online ahead of print, $2020 \mathrm{Jul}$ 7]. Int J Cancer. 2020. https://doi.org/10.1002/ijc.33203.

2. West $\mathrm{H}$, Jin JO. Performance status in patients with cancer. JAMA Oncol. 2015;1:998.

3. Penel N, Glabbeke MV, Mathoulin-Pelissier S, et al. Performance status is the most powerful risk factor for early death among patients with advanced soft tissue sarcoma: the European Organisation for Research and Treatment of Cancer-Soft Tissue and Bone Sarcoma Group (STBSG) and French Sarcoma Group (FSG) study. Br J Cancer. 2011;104(10):1544-1550.

4. Giobbie-Hurder A, Gelber RD, Regan MM. Challenges of guaranteetime bias. J Clin Oncol. 2013;31:2963-2969. 